\title{
Occupational safety and health education and training: an innovative format and experience
}

\author{
Sergio Iavicoli ${ }^{1}$, Emma Pietrafesa ${ }^{1}$, Agnese Martini ${ }^{1}$, Rosalba Simeone ${ }^{2}$, Antonella \\ Polimeni $^{3}$
}

${ }^{1}$ Department of Occupational and Environmental Medicine, Epidemiology and Hygiene, INAL - The National Institute for Insurance against Accidents at Work, Rome, Italy, ${ }^{2}$ Department of Oral and Maxillo-Facial Sciences, Sapienza University of Rome, Rome, Italy, ${ }^{3}$ Faculty of Medicine and Dentistry, Sapienza University of Rome, Rome, Italy.

\begin{abstract}
Health begins at home and in community where people live and work, in fact, the World Health Organisation (WHO) defines health as 'a state of complete physical, mental and social well-being'. Experts and professionals, of all sectors and specialities, need to take account the Occupational Safety and Health (OSH) in all aspects of their working lives. Mainstreaming OSH into education concerns integrating one policy area - OSH - into another education. This study started from a first analysis of an international and national OSH training offer, in which some critical aspects emerged: there are mostly sectoral training courses, qualifying some prevention actor roles, most linked to traditional risks, and primarily focused on the safety aspects rather than the health ones. The current study is related to an innovative format and experience for an integrated management of OSH in the evolution of the world of work. The concept was born from the need to train new professionals figures when the rapid demographic changes and technological innovation are changing the working world and therefore also the required skills to prevention actors. A first test has been conducted on 26 students who attended the first edition of this innovative training.
\end{abstract}

Keywords: education, training; occupational safety; health; university courses. 


\section{Introduction}

The World Health Organisation defines health as 'a state of complete physical, mental and social well-being' (WHO, 1948). Health everywhere around the world begins at home and in community where people live and work.

Work-related mortality accounted for 5\% of the global total deaths (Global Burden of Disease Study, 2015). Every 15 seconds, a worker dies due to an accident at work or an occupational disease, in fact, the International Labor Organization (ILO) estimates that approximately 2.78 million women and men in worldwide succumb to accidents or workrelated illnesses: over 6000 deaths per day. In this scenario, education and training play a fundamental role, since beyond the specific regulatory interventions and the obligations connected to them. Developing, disseminating and concretizing a culture of prevention for $\mathrm{OSH}$, it remains today a stimulating request that involves stakeholders interested in the enhancement of "human capital" as the main resource for the realization of a concrete and continuous process of improving the quality of life, including the working one.

The OSH Community strategy 2007-2012 considered the culture of prevention to be an important area of action; according to this strategy, risk education should be included in all levels of education, University too (Copsey et al., 2010). According to the latest $\mathrm{OSH}$ policy, risk education is become more important due to rapid development of the society, new technologies, globalization and changed demographic processes (Reinhold et al., 2013). An important step in training professionalization and education was taken with the establishment of the first University courses in safety, during the 1970s, in some EU countries (Germany, France, United Kingdom and Belgium). Studies conducted by the International Social Security Association (ISSA) Section on Education and Training for Prevention, and later by the European Network of Safety and Health Professional Organisation (ENSHPO) have shown that there is a wide disparity between the definitions of safety professionals and their role and training in the different EU countries. A first point to an European harmonization was boosted by the initiative of the ISSA Safety Training Section, later taken over by ENSHPO, which documented the regulatory schemes and the range of roles and competences of safety experts and professionals across Europe. The European project (2010-2012), so called EUSafe, was taking a further step to develop the standards into exemplary role and task descriptions, learning objectives and teaching protocols which could be used to stimulate further training initiatives and lead to further harmonization of training requirements. Its objectives are to better underpin the definitions of the role and training needs of safety experts and professionals across Europe. This European development has now combined with a parallel development internationally under INSHPO bringing together North American and Asia Pacific countries to share and learn from each other's certification and accreditation systems (Swuste \& van Dijk, 2018). 


\section{Methods}

The analysis and reflections process about good practices, and learned lessons was carried out based on the exchange among the institutional leaders, the team of teachers and students and was complemented by the researcher of these advisory services. First, an analysis of the international and national literature about the OSH available courses has been done, than the study focused on the changes in the world of work, in order to desing and develop a proposal for an innovative training format. The analysis has been carried out into the internet through different search engines using the following keywords: "university", "occupational safety", "occupational health", "master", "post-graduate course". The structure of the individual courses was deepened through the direct internet links. The analysis of the international educational offer followed this kind of methodology, while national research was developed on the study of the educational offer of the single Universities. The last step of the study was to delivery and evaluate the training format. The assessment of learning process was done through an anonymous questionnaire at the end of the training. The 26 students of the first edition of the Master (14 men and 12 women, aged 28-56yr) completed the questionnaire. The first edition of the Master course took place from February 2018 to December 2019. This first edition was attended by students came only from Italy. The 10 item self-administered questionnaire aim to assess the quality of the purpose, content, timing and mode, using a 5-points Likert scale.

\section{Results}

\subsection{Analysis of international and national University training on occupational safety and health $(\mathrm{OSH})$}

The OSH international offer provides a typically two-year period training. In several cases the period of the Master courses is linked to a frequency mode: such as part-time (two years) or full-time (one year). The training is varied and consists of classroom lessons, distance and online learning, visits to the companies, residential training at University campuses. The main subjects covered are: health and safety at work, occupational hygiene, occupational psychology, ergonomics, epidemiology, risk management and health promotion.

An analysis via internet of the training offer, existing at national level of University courses in the OSH field provided in the last ten years, shows that, since the 90's in Italy, there has been a growing training offer on OSH subjects, mainly due to an increase in the educational/training needs for prevention actors, due also to the implementation of the regulatory framework. In this context, the University has contributed with post-graduate training offers (1st and 2nd level Masters and Advanced Training courses). At the national level 19 Master's courses were counted and in particular 8 of them were dedicated to the 2 th 
level of University degree and 11 to the first one. Almost all of them involved collaboration with other Universities, professional scientific associations, Institutions of the National Health Service, as well as the National Institute for Insurance against Accidents at Work (INAIL). The Master courses generally have a duration of one year, with a teaching commitment about 1500 hours totally. Courses are provided through frontal teaching activities and other forms of guided study, distance learning interactive activity (FADeLearning mode) or by providing both methods (Blended learning). In some cases the Master's students have obtained the qualifications required by law to perform certain professions such as the person in charge of prevention and protection service (RSPP and ASPP) pursuant to art. 32 of Legislative Decree 81/08.

\subsection{Analysis of the changes in the world of work and the training needs for the development of the Master course}

In the OSH policy, one of the main goal of the "European Union Strategic Framework 2014-2020" is to face demographic change by requiring adequate working conditions, in order to guarantee a sustainable working life and an active and healthy ageing. In fact, it is necessary to consider the existence of a gap between life expectancy and employment expectation, mainly due to deterioration of health and early withdrawal from work due to illness and/or injury. Moreover, the current scenario of the working world is characterized by important technological innovations - robotics, smart working, key enabling technologies (KETs) - that will profoundly transform industrial processes, offering great prospects for development in terms of productivity and plant quality. This landscape of the future of work will entail further complexities to manage and set in with a view to prevention, in order to increase the sustainability of work, including: new forms of work organisation and new risks, the need to face important changes in the work management (e.g. flexible forms of work, new forms of work organisation, psychosocial aspects), the need to understand the influence of age-related changes in work capacity, particularly in the case of specific strenuous jobs, as well as the impacts on the obsolescence of skills in hightech jobs. Therefore, there is a need to train new specialized figures, able to respond to the changing of the working world through the integrated risk management, based on a multidisciplinary and participatory approach, which integrates different aspects and specific competences of different disciplinary areas related to the issue of health and safety at work, such as occupational health, public health, occupational psychology, health psychology, psychometrics, management and ergonomics. This approach, however widely expected, was still lacking in Italy due to the absence of a specific recognized interdisciplinary training, which integrates skills in the field of occupational health prevention, including those related to aspects of a psychosocial and organisational nature, with the skills required in the field of safety in the workplaces. 


\subsection{Design and development of the Master in integrated Health and Safety Management in the evolution of the working world}

Public and private sector organizations are increasingly concerned with improving occupational safety and health. The rapid changes in the world of work, combined with the persistence of unsafe or environmentally hazardous working conditions, have served to focus attention on the need to create safe, healthy working environments and OSH professionals with the right skills. According to the analysis of the national training offer up to date, it emerges that the courses activated were mainly conditioned by the formal requirements defined and established by current legislation for the recognition of the role of worker prevention and protection professionals, leaving little room for the development of knowledge and skills related to innovation and work changes. Becoming an occupational safety and health professional requires multidisciplinary training with a broad-based educational background, including specialized knowledge of the medicine, law, chemistry, engineering and social sciences together with an understanding of the principles and concepts of management. The national and international training offers put in evidence some critical aspects: these are mostly sectoral training courses, qualifying some prevention actor roles, most linked to traditional risks, and primarily focused on the safety aspects rather than the health ones. A special consideration deserves the advantages offered by University training programs that can open up opportunities for in-depth study and professional updating to existing prevention figures, on innovative and emerging issues.

The biennial training course in "Integrated management of health and safety in the evolution of the working world" is promoted by Sapienza University of Rome, involving four Faculties (the two-ones of Medicine, Civil and Industrial Engineering and Law) and the research departments and the central and technical facilities of INAIL. The concept was born from the need to train new professional figures when the rapid demographic changes and technological innovation are changing the world of work and therefore also the required skills to prevention actors. The Master has innovative features both for the topics being discussed and for its structure aimed at a multidisciplinary application and professionalization approach. The two-year Master's program has allowed the networking of training, innovation and research, namely INAIL, University and the productive world in order to respond concretely to the increasingly complex challenges of the working world, to grasp all the opportunities in terms of development, research, innovation and employability. Many leading companies in the field of nanotechnology and in the high automation industrial sector and the enabling technologies of Industry 4.0, have chosen to play an active role within the innovative INAIL-Sapienza path, offering to participants the possibility of innovative internships projects. The planning of the internship projects was carried out with a multidisciplinary, innovative and integrated approach, with particular regard to the "prevention through design". Among the most interesting activities carried out 
in the first edition these areas were studied in deep: ergonomic assessments aimed at reducing biomechanical overload in the industrial sector; availability, integration and knowledge of data on work-related accidents to develop "road safety" and sustainable mobility methodologies and paths; improvement of safety management systems and application of technology to prevent accidents at work through a proactive and integrated approach; analysis and correlation models between natural and incidental health and safety events aimed at the well-being and global protection of the worker, according to the OneHealth conception.

The course has been designed to a limited number (maximum of 30 students), in order to make possible the carrying out of high quality and interactive of the didactic activities. It has lasted two years with a teaching commitment of 1,500 hours overall, of which at least 350 hours reserved to lectures of frontal teaching and 125 hours dedicated to the final exam. The sum of the aforementioned activities corresponds to the acquisition of 60 University credits (CFU) in addition to the credits already acquired for the course of study necessary to access to the course. Each CFU corresponds to 25 hours of study divided into the various training activities (Table 1).

\subsection{Assessment of the innovative training format}

The main results of the evaluation of the first edition of the Master are summarized in the following table (Table 2).

In summary, the analysis shows the following results for the individual teaching modules: the mean value of the Module 1 is 4.2 (SD 0.8; Likert scale 1 till 5), the mean value of the Module 2 is 4.3 (SD 0.8; Likert scale 1 till 5), the mean value of the Module 3 is 4.0 (SD 1.3; Likert scale 1 till 5). 
Table 1. Master Structure

\section{Course Structure}

Module 1.General aspects of integrated risk management and regulatory framework

The module is aimed at the development of knowledge and general skills in the following areas: occupational health, legal, insurance, statistics-epidemiology, psychosocial, risk assessment and management, safety at work, able to characterize, manage and develop solutions for an integrated approach to risks with particular regard to the contemporary labor market. Qualification of Head of the Prevention and Protection Service (RSPP) or of Operator of the Prevention and Protection Service (ASPP)

Module 2. Specialized training focused on the change in the working world

The module is aimed at developing skills, knowledge and experiences in areas closely linked to the change in the working world, developed through highly profiled activities towards innovative aspects of health and safety related in particular to demographic changes and technological evolution.

\section{Module 3. Integrated risk management}

The module is aimed at developing skills and experiences aimed at identifying innovative measures and techniques for integrated risk assessment and management. The module also provides for further activities, including the training internship in partner organizations rooted in the working/production contexts and in close contact with the health and safety sector and/or at the Sapienza University and INAIL laboratories.

Seminar activities related to the themes of the training path, initiatives and training days in collaboration with the partner bodies of the Master.

\begin{tabular}{lc}
\hline Final Exam & 5 \\
\hline Total & 60 \\
\hline
\end{tabular}

Table 2. Assessment of the first edition of the Master

\begin{tabular}{|c|c|c|c|c|c|c|c|c|}
\hline & \multicolumn{3}{|c|}{ Module 1} & \multicolumn{3}{|c|}{ Module 2} & \multicolumn{2}{|c|}{ Module 3} \\
\hline & $\begin{array}{l}\text { Education } \\
\text {-training } \\
\text { (content } \\
\text { and mode) }\end{array}$ & $\begin{array}{c}\text { Teaching } \\
\text { quality }\end{array}$ & $\begin{array}{l}\text { Tutoring } \\
\text { quality }\end{array}$ & $\begin{array}{l}\text { Education } \\
\text { - training } \\
\text { (content } \\
\text { and mode) }\end{array}$ & $\begin{array}{c}\text { Teaching } \\
\text { quality }\end{array}$ & $\begin{array}{l}\text { Tutoring } \\
\text { quality }\end{array}$ & $\begin{array}{c}\text { Training } \\
\text { internship } \\
\text { (content, } \\
\text { mode, } \\
\text { timing) }\end{array}$ & $\begin{array}{c}\text { Tutoring } \\
\text { quality }\end{array}$ \\
\hline $\begin{array}{l}\text { Mean } \\
\text { value }\end{array}$ & 3.9 & 4.3 & 4.4 & 4.2 & 4.4 & 4.4 & 4.0 & 4.1 \\
\hline SD & 1.0 & 0.8 & 0.6 & 0.9 & 1.1 & 0.7 & 1.3 & 1.4 \\
\hline
\end{tabular}




\section{Conclusions}

All the actors involved in training and work agree that it is in training process where we should acquire knowledge, develops skills and internalize risk identification and prevention behaviours at work. This Master course, which was promoted for the first time in Italy, has been integrated with advanced training courses that the student during the second year can choose in a modular way. This two-year training course presents innovative features not only for its structure, but also for the topics under discussion focused on aspects related in particular to changes in the world of work (e.g. demographic changes) and technological innovation (e.g. nanotechnologies, industry 4.0, enabling technologies, IoT, process digitalization, automation, robotics, smart working, digital transformation). The innovative features related to its structure, was obtained through the integration of two didactics training offers, the Master, a third cycle, scientific and high specialized course for lifelong and recurrent training, with the advanced training courses (CAF) for a postgraduate specialization or specialist studies.

As the epistemologist Piaget highlighted the collaboration between different disciplines or heterogeneous sectors of the same science, provided real interactions, reciprocity of exchanges, such as to determine mutual enrichments. With this inter-multidisciplinary perspective, the training path of the Master Sapienza-Inail was organized and observed aimed at allowing learners and teachers an innovative experience of an interactive process guaranteed by the loan and the methodological exchange of the various disciplines that carried out the cultural-scientific enrichment and the creation of a transdisciplinary system in $\mathrm{OSH}$ area.

The present format seems to be a good practice for the integration of research, educationtraining, work and innovation, as students refered.

\section{References}

Copsey, S., Debruyne, M., Eeckelaert, L., Malmelin, J., Salminen, S., Buffet, M. A. \& Bollmann, U. (2010). Mainstreaming occupational safety and health into university education. Publications Office of the European Union.

European Agency for Safety and Health at Work. (2010). Mainstreaming OSH into Business Management.

Hämäläinen P., Takala J., Tan Boon Kiat. Global estimates of occupational accidents and work-related illnesses. (2017). Published in September 2017 by Workplace Safety and Health Institute. ISBN: 9789811148446. www.icohweb.org

Reinhold, K., Siirak, V., \& Tint, P. (2014). The development of higher education in occupational health and safety in Estonia and selected EU countries. Procedia-Social and Behavioral Sciences, 143, 52-56. 
Swuste P., van Dijk F. (2018). Evaluation of quality of academic safety, health and environment education. ENETOSH-European network education and training in occupational safety and health. Fact sheet 04.

Wang, H., Naghavi, M., Allen, C., Barber, R. M., Bhutta, Z. A., Carter, A., \& Coggeshall, M. (2016). Global, regional, and national life expectancy, all-cause mortality, and cause-specific mortality for 249 causes of death, 1980-2015: a systematic analysis for the Global Burden of Disease Study 2015. Lancet, 388(10053), 1459-1544.

World Health Organization. (1995). Constitution of the world health organization. 